\title{
Cauchy problems for noncoercive Hamilton-Jacobi-Isaacs equations with discontinuous coefficients
}

\author{
Cecilia De ZAN \\ Dipartimento di Matematica Pura e Applicata, \\ Università di Padova, via Trieste 63, 35121 Padova, Italy \\ E-mail: cdezan@math.unipd.it \\ Pierpaolo Soravia \\ Dipartimento di Matematica Pura e Applicata, \\ Università di Padova, via Trieste 63, 35121 Padova, Italy \\ E-mail: soravia@math.unipd.it
}

[Received 30 October 2009 and in revised form 23 July 2010]

\begin{abstract}
We study the Cauchy problem for a homogeneous and not necessarily coercive Hamilton-JacobiIsaacs equation with an $x$-dependent, piecewise continuous coefficient. We prove that under suitable assumptions there exists a unique and continuous viscosity solution. The result applies in particular to the Carnot-Carathéodory eikonal equation with discontinuous refraction index of a family of vector fields satisfying the Hörmander condition. Our results are also of interest in connection with geometric flows with discontinuous velocity in anisotropic media with a non-euclidian ambient space.
\end{abstract}

2010 Mathematics Subject Classification: Primary 35D40; Secondary 35F21, 35F25, 49L20.

\section{Introduction}

We consider the Cauchy problem for a Hamilton-Jacobi equation

$$
\left\{\begin{array}{l}
u_{t}(x, t)+f(x) H(x, D u(x, t))=0, \quad(x, t) \in \mathbb{R}^{n} \times(0,+\infty), \\
u(x, 0)=u_{0}(x) \in C\left(\mathbb{R}^{n}\right),
\end{array}\right.
$$

where the continuous Hamiltonian $H: \mathbb{R}^{n} \times \mathbb{R}^{n} \rightarrow \mathbb{R}$ and the function $f: \mathbb{R}^{n} \rightarrow[0,+\infty)$ satisfy

$H(x, \cdot)$ is positively 1 -homogeneous,

$f$ is bounded, piecewise Lipschitz continuous across Lipschitz hypersurfaces,

$H \geqslant 0, \quad f(x) \geqslant c_{0}>0$.

When discontinuities in the $x$ variable are present, one can expect neither continuity of the viscosity solutions, nor their uniqueness, in general. In this paper we will give sufficient conditions for the existence and uniqueness of a continuous solution of (1.1). We remark that $H(x, \cdot)$ is not necessarily convex and the Hamiltonian is not assumed to be coercive: we allow $\liminf _{|p| \rightarrow \infty} H(x, p)=0$ (or, equivalently for homogeneous $H(x, \cdot), \inf _{|p|=1} H(x, p)=0$ ) for all $x \in \mathbb{R}^{n}$. We prove a general comparison principle for (discontinuous) super/subsolutions of (1.1), subject to a priori continuity along suitable transversal directions to the set $\Gamma$ of the space discontinuities of $f=f(x)$. In order to prove existence of viscosity solutions that satisfy 
the continuity properties needed to apply our comparison principle, we use a control-theoretic representation of solutions. We rewrite the Hamiltonian as an Isaacs equation in differential games, by putting

$$
H(x, p)=\min _{b \in B} \max _{a \in A}\{-g(x, a, b) \cdot p\},
$$

where $A, B$ are compact metric spaces and $g: \mathbb{R}^{n} \times A \times B \rightarrow \mathbb{R}^{n}$ is a controlled vector field, and we require controllability of the dynamics corresponding to $g$ transversally to $\Gamma$. We also prove stability of our unique solution with respect to approximations of $f$ from below and above with continuous functions.

Problem 1.1 can be viewed as the Bellman-Isaacs equation of an optimal control problem or differential game of Mayer type with a discontinuous vector field,

$$
u_{t}+\min _{b \in B} \max _{a \in A}\{-f(x) g(x, a, b) \cdot D u\}=0,
$$

the vector field being $f(x) g(x, a, b)$. This is a very interesting problem in itself in control theory, and the representation of viscosity solutions as value functions is an almost completely open problem. Here however we strongly limit the structure of the discontinuities of the vector field.

Problem (1.1) also appears in the study of the refraction phenomenon in anisotropic media with a discontinuous refraction index. Related to this is the subject of weak front evolution with discontinuous normal velocity. By homogeneity of $H(x, \cdot)$ one can easily observe that if $u$ solves the pde in $(1.1)$ and $\psi: \mathbb{R} \rightarrow \mathbb{R}$ is smooth and increasing, then also $\psi(u)$ solves the same HJ equation. Hence the equation is called geometric. As a consequence, if $u_{0}^{1}$ and $u_{0}^{2}$ are two initial conditions such that

$$
\Lambda_{0}=\left\{x: u_{0}^{1}(x)=0\right\}=\left\{x: u_{0}^{2}(x)=0\right\},
$$

is a closed hypersurface and $u^{1}, u^{2}$ are the corresponding solutions of the Cauchy problem for the HJ equation, then one shows that

$$
\left\{x: u^{1}(x, t)=0\right\}=\Lambda_{t}=\left\{x: u^{2}(x, t)=0\right\}
$$

for $t>0$ and we can define the family $\left(\Lambda_{t}\right)_{t}$ as the geometric flow of the hypersurface $\Lambda_{0}$ with discontinuous normal velocity $1 / f$ in the anisotropic medium. This is the key observation of the level set method for weak front propagation, introduced by Osher-Sethian [17]. For the mathematical analysis of the level set method via viscosity solutions, the reader is referred to the book by Giga [13], where the above uniqueness in the level set approach is also discussed in detail for general, second order, geometric equations. We will use the well posedness of (1.1) to study the limit of reaction-diffusion equations generating geometric flows with discontinuous normal velocity in a forthcoming paper [10].

Degenerate Hamiltonians, that we put our emphasis on, appear in particular in media subject to non-euclidian metrics. Our main examples are metric structures of Carnot-Carathéodory (or CC) type. In this case the Hamiltonian $H$ (say that $H(x, \cdot)$ is now convex) has the form $H(x, p)=$ $\tilde{H}\left(x, \sigma^{t}(x) p\right)$, where $\tilde{H}$ is coercive, and $\sigma$ is an $n \times m$ matrix (here $m<n$ ) whose columns represent a family of vector fields. Our results include in particular the CC eikonal equation, where $H(x, p)=\left|\sigma^{t}(x) p\right|$. The study of CC or subelliptic equations is a field of growing interest (see e.g. the book by Bonfiglioli-Lanconelli-Uguzzoni [3] and the references therein) and has potentially wide applications. For instance, recent models of image perception of the visual cortex are based on sub-Riemannian metrics (see e.g. Citti-Sarti [8]). 
The theory of viscosity solutions for Hamilton-Jacobi equations with discontinuous coefficients has by now a rather long history. The problem was first studied by Ishii [15] who defined viscosity solutions for Hamiltonians discontinuous in the variables $(t, u)$. In the case that discontinuities of the Hamiltonian concern the state variable $x$, the problem is quite interesting and more delicate. In order to have well posed problems, one either needs to have some strong structure of the Hamiltonian and to strengthen the classical notion of viscosity solution, or to strongly limit the class of discontinuities allowed. Within the first group of papers, we recall the work on uniformly elliptic/parabolic pdes by Jensen [16] and by Caffarelli-Crandall-Kocan-Święch [4] (see also the references therein). Also, for first order, convex and coercive Hamiltonians (i.e. $H(x, p) \rightarrow+\infty$ as $|p| \rightarrow \infty)$ uniqueness results have been obtained by Camilli-Siconolfi [6] and in the case of geometric equations by Camilli [5]. Other studies on more general classes of equations, in particular degenerate and noncoercive Hamiltonians, using the standard notion of viscosity solution, are limited to piecewise continuous coefficients. We recall the work of one of the present authors on the characterization of uniqueness of viscosity solutions [20], existence and uniqueness results for the stationary eikonal equation [21], and general uniqueness results for degenerate elliptic equations [22]; see however also the references therein for additional work on the subject. More recently, uniqueness results for Lipschitz continuous solutions of (1.1) with methods different than ours have been obtained by Chen-Hu [7] for nonconvex but radial and $x$-independent $H$, with new sets of assumptions on the discontinuous coefficient.

The paper is organized as follows. In Section 2 we present a few preliminaries and an example showing that viscosity solutions of (1.1) can be discontinuous. In Section 3 we prove the comparison principle. In Section 4 we give our main existence and uniqueness results.

\section{Main assumptions and examples}

We now recall what we mean by a locally bounded viscosity solution $u: \mathbb{R}^{n} \times(0,+\infty) \rightarrow \mathbb{R}$ of the pde in [1.1]. Observe that $f, H \geqslant 0$, so following Ishii [15] we can define viscosity solutions of

$$
u_{t}(x, t)+f(x) H(x, D u(x, t))=0
$$

by checking the two differential inequalities

$$
\begin{aligned}
& u_{t}(x, t)+f_{*}(x) H(x, D u(x, t)) \leqslant 0, \\
& u_{t}(x, t)+f^{*}(x) H(x, D u(x, t)) \geqslant 0,
\end{aligned}
$$

in the standard viscosity sense (see [9, 1]). Here $f^{*}(\hat{x})=\lim _{r \rightarrow 0^{+}} \sup _{x \in B(\hat{x}, r)} f(x)$ is the upper semicontinuous envelope, and the lower semicontinuous envelope $f_{*}$ is defined analogously. For instance, to say that $u$ is a subsolution means that whenever $\varphi \in C^{1}\left(\mathbb{R}^{n} \times(0,+\infty)\right)$ and $u^{*}-\varphi$ has a local maximum point at $\left(x_{0}, t_{0}\right)$, then

$$
\varphi_{t}\left(x_{0}, t_{0}\right)+f_{*}\left(x_{0}\right) H\left(x_{0}, D \varphi\left(x_{0}, t_{0}\right)\right) \leqslant 0 .
$$

A locally bounded function $u: \mathbb{R}^{n} \times[0,+\infty)$ will be a (discontinuous) solution of (1.1) if moreover it is continuous at the points of $\left\{(x, 0): x \in \mathbb{R}^{n}\right\}$ and $u(x, 0)=u_{0}(x)$.

In the classical theory of viscosity solutions, when a HJ pde satisfies the comparison principle between upper semicontinuous subsolutions and lower semicontinuous supersolutions, a byproduct is the continuity of solutions. One of the difficulties that we encounter in our analysis is due to the 
fact that solutions may be discontinuous even for continuous initial conditions, as the next example shows. We will however characterize unique solutions only when they are continuous.

EXAMPLE 2.1 Consider the Cauchy problem

$$
\left\{\begin{array}{l}
u_{t}(x, y, t)+f(x)\left|u_{y}(x, y, t)\right|=0 \\
u(x, y, 0)=x^{2}+y
\end{array}\right.
$$

where $f(x)=1$ for $x<0$, and $f(x)=2$ for $x>0$. It is then easy to check that a viscosity solution is

$$
u(x, y, t)= \begin{cases}x^{2}+y-t, & x<0, \\ x^{2}+y-2 t, & x>0,\end{cases}
$$

which is clearly discontinuous on $\{(0, y, t): t>0\}$. Moreover in this example the geometric flow of the initial hypersurface

$$
\Lambda_{0}=\left\{(x, y): x^{2}+y=0\right\}
$$

breaks up for $t>0$ as

$$
\Lambda_{t}=\left\{(x, y): x^{2}+y=t, x<0\right\} \cup\left\{(x, y): x^{2}+y=2 t, x>0\right\} .
$$

Thus discontinuous solutions do not provide a meaningful propagating front.

As we mentioned in (1.2), we will consider only special discontinuities in the coefficient $f$. We now make this precise.

DEFINITION 2.2 We say that $f: \mathbb{R}^{n} \rightarrow[0,+\infty)$ is a piecewise Lipschitz continuous function if its discontinuity set $\Gamma \subset \mathbb{R}^{n}$ is a finite union of Lipschitz hypersurfaces with the following properties. For any $\hat{x} \in \Gamma$ there is $\hat{r}>0$ such that we can partition

$$
B(\hat{x}, \hat{r})=\Omega_{\hat{x}}^{+} \cup \Omega_{\hat{x}}^{-} \cup(\Gamma \cap B(\hat{x}, \hat{r})),
$$

where $\Omega_{\hat{x}}^{ \pm}$are nonempty, open, connected (locally, the two sides of $\Gamma$ ). Moreover, $\inf _{\Omega_{\hat{x}}^{+}} f>$ $\sup _{\Omega_{\hat{x}}^{-}} f ; f$ is locally Lipschitz continuous in $\mathbb{R}^{n} \backslash \Gamma ; f$ has a Lipschitz continuous extension in $\Omega_{\hat{x}}^{+} \cup\left(\Gamma \cap B(\hat{x}, \hat{r})\right.$ ) (called $f^{*}$ ), and $\Omega_{\hat{x}}^{-} \cup\left(\Gamma \cap B(\hat{x}, \hat{r})\right.$ ) (called $f_{*}$ ); for all $x \in \Gamma$ we have $f(x) \in\left[f_{*}(x), f^{*}(x)\right]$. Below, we usually drop the subscript $\hat{x}$ in $\Omega_{\hat{x}}^{ \pm}$.

REMARK 2.3 When $f$ is piecewise continuous and $\Gamma$ is the union of disjoint Lipschitz hypersurfaces, at every $\hat{x} \in \Gamma$ we can always find unit vectors $\eta^{+}, \eta^{-} \in \mathbb{R}^{n}$ pointing into $\Omega^{+}, \Omega^{-}$ respectively (transversal to $\Gamma$ ). This means that for some $c, h>0$ we have $B\left(y+t \eta^{+}, t c\right) \subset \Omega^{+}$ for all $y \in B(\hat{x}, h) \cap \bar{\Omega}^{+}$and $t \in(0, c)$ (see [2]). Similarly for $\eta^{-}$.

We now introduce some technical assumptions on the Hamiltonian that we need in the following. For every $K \subset \subset \mathbb{R}^{n}$,

$$
|H(x, p)-H(x, q)| \leqslant \omega_{K}(|p-q|) \quad \forall x \in K, \forall p, q \in \mathbb{R}^{n},
$$

where $\omega_{K}$ is a modulus of continuity. 
Given $(\hat{x}, \hat{t}) \in \mathbb{R}^{n} \times(0,+\infty)$ and for $\eta=\eta_{\hat{x}}^{-}$any unit vector pointing into $\Omega^{-}$if $\hat{x} \in \Gamma$, or $\eta=0$ if $\hat{x} \in \mathbb{R}^{n} \backslash \Gamma$, we have

$$
H(x, \alpha(x-y)+\sqrt{\alpha} \eta)-H(y, \alpha(x-y)+\sqrt{\alpha} \eta) \geqslant-\omega_{\hat{x}}\left(|x-y|+\alpha\left|x-y+\frac{1}{\sqrt{\alpha}} \eta\right|^{2}\right)
$$

for all $x, y \in B(\hat{x}, s), \alpha>0$, if $s>0$ is sufficiently small.

Given $(\hat{x}, \hat{t}) \in \mathbb{R}^{n} \times(0,+\infty)$ and for $\eta=\eta_{\hat{x}}^{+}$any unit vector pointing into $\Omega^{+}$if $\hat{x} \in \Gamma$, or $\eta=0$ if $\hat{x} \in \mathbb{R}^{n} \backslash \Gamma$, we have

$$
H(x, \alpha(x-y)-\sqrt{\alpha} \eta)-H(y, \alpha(x-y)-\sqrt{\alpha} \eta) \geqslant-\omega_{\hat{x}}\left(|x-y|+\alpha\left|x-y-\frac{1}{\sqrt{\alpha}} \eta\right|^{2}\right)
$$

for all $x, y \in B(\hat{x}, s)$ and $\alpha>0$, if $s>0$ is sufficiently small.

REMARK 2.4 It is easy to check that the assumptions (2.2), (2.3) are satisfied in particular for Hamiltonians of the form 1.3 if $A, B \subset \mathbb{R}^{k}$ are compact and $g: \mathbb{R}^{n} \times A \times B \rightarrow \mathbb{R}^{n}$ is bounded, continuous and such that

$$
|g(x, a, b)| \leqslant M_{g}, \quad|g(x, a, b)-g(y, a, b)| \leqslant L_{g}|x-y|
$$

for all $x, y \in \mathbb{R}^{n}, a \in A, b \in B$.

We recall moreover that by Evans-Souganidis [12], any 1-homogeneous Hamiltonian which is Lipschitz continuous in $\mathbb{R}^{n} \times \mathbb{R}^{n}$ can be represented in the form $[1.3$, where moreover min and max can be interchanged, which is known as the Isaacs condition.

\section{Comparison principle for the $\mathrm{HJ}$ equation}

The first result of this section is the following comparison principle for solutions in a finite timeinterval. Since the equation has a discontinuous term, it requires some a priori continuity of the functions to be compared. Such continuity properties will be better analyzed in the next section.

Theorem 3.1 Let $T \in(0,+\infty]$ and assume $\left[2.1-2.3\right.$ and $(1.2)$. Let $u, v: \mathbb{R}^{n} \times[0, T] \rightarrow \mathbb{R}$ be respectively an upper semicontinuous subsolution and a lower semicontinuous supersolution of the HJ equation

$$
w_{t}(x, t)+f(x) H(x, D w(x, t))=0, \quad(x, t) \in \mathbb{R}^{n} \times(0, T),
$$

such that $u(x, 0) \leqslant v(x, 0)$ and, if $T<+\infty$,

$$
u(x, T)=\limsup _{(y, s) \rightarrow\left(x, T^{-}\right)} u(y, s), \quad v(x, T)=\liminf _{(y, s) \rightarrow\left(x, T^{-}\right)} v(y, s) .
$$

Suppose moreover that for all $(\hat{x}, \hat{t}) \in \Gamma \times(0, T]$ we can find sequences $\varepsilon_{k} \rightarrow 0^{+}, \sigma_{\varepsilon_{k}} \rightarrow 0$, and $p^{\varepsilon_{k}} \in \mathbb{R}^{n}$ with $\left|p^{\varepsilon_{k}}\right| \rightarrow 0^{+}$such that $\sigma_{\varepsilon_{k}} \leqslant 0$ if $\hat{t}=T$, and either

$$
\lim _{k \rightarrow+\infty} u\left(\hat{x}+\varepsilon_{k} \eta^{+}+\varepsilon_{k} p^{\varepsilon_{k}}, \hat{t}+\sigma_{\varepsilon_{k}}\right)=u(\hat{x}, \hat{t})
$$


or

$$
\lim _{k \rightarrow+\infty} v\left(\hat{x}+\varepsilon_{k} \eta^{-}+\varepsilon_{k} p^{\varepsilon_{k}}, \hat{t}+\sigma_{\varepsilon_{k}}\right)=v(\hat{x}, \hat{t}),
$$

where $\eta^{+}, \eta^{-}$are unit vectors pointing into $\Omega^{+}, \Omega^{-}$, respectively with the notation of Definition 2.2 and Remark 2.3 . Then $u \leqslant v$ in $\mathbb{R}^{n} \times[0, T]$.

REMARK 3.2 The coefficient $f$ has discontinuity set $\Gamma \times[0, T]$ in the $(x, t)$ space. In this sense the continuity of the functions $u, v$ is required along families of points $\left(x_{\varepsilon}, t_{\varepsilon}\right)=\left(\hat{x}+\varepsilon \eta^{ \pm}+\varepsilon p^{\varepsilon}, \hat{t}+\sigma_{\varepsilon}\right)$ such that $\left(x_{\varepsilon}-\hat{x}\right) / \varepsilon \rightarrow \eta^{ \pm}$(transversal to $\Gamma$ ), but the way $\sigma_{\varepsilon}$ tends to 0 is not prescribed. Actually, as we show in Section 4, many interesting cases are when $\varepsilon=o\left(\sigma_{\varepsilon}\right)$. In this case $\left(x_{\varepsilon}, t_{\varepsilon}\right)$ tend to $(\hat{x}, \hat{t}) \in \Gamma \times[0, T]$ in a tangential fashion. For this reason the comparison principle above is not a direct consequence of the general result by one of the authors in [22], although the method of proof we use is similar.

In the proof below we need to use a standard lemma whose proof is for instance contained in [1].

Lemma 3.3 Suppose that $f, H \geqslant 0$ and $H$ is continuous. Let $T \in(0,+\infty)$. Assume that $u$ (resp. $v$ ) : $\mathbb{R}^{n} \times(0, T) \rightarrow \mathbb{R}$ is an upper semicontinuous viscosity subsolution (resp. lower semicontinuous supersolution) of

$$
u_{t}(x, t)+f(x, t) H(x, D u(x, t))=0, \quad(x, t) \in \mathbb{R}^{n} \times(0, T),
$$

and that

$$
u(x, T):=\limsup _{(y, s) \rightarrow\left(x, T^{-}\right)} u(y, s) \quad\left(\operatorname{resp} . v(x, T):=\liminf _{(y, s) \rightarrow\left(x, T^{-}\right)} v(y, s)\right) .
$$

Then $u$ (resp. $v$ ) is a subsolution (resp. supersolution) of

$$
u_{t}(x, t)+f(x, t) H(x, D u(x, t))=0 \quad \text { in } \mathbb{R}^{n} \times(0, T] .
$$

REMARK 3.4 We recall that in order to check whether (3.3) is satisfied by $u$ as a viscosity subsolution at a point of the form $(\hat{x}, T)$, we have to check local maxima of $u-\Phi, \Phi \in$ $C^{1}\left(\mathbb{R}^{n} \times(0, T]\right)$, relative to $\mathbb{R}^{n} \times(0, T]$.

Proof of Lemma 3.3 Since our equation is invariant under an increasing change of the dependent variable, it is not restrictive to suppose that $u, v$ are bounded.

Assume now towards a contradiction that there is $\left(x_{0}, t_{0}\right) \in \mathbb{R}^{n} \times(0, T]$ such that

$$
u\left(x_{0}, t_{0}\right)-v\left(x_{0}, t_{0}\right)=A>0 .
$$

We set $x_{0}=0$ for simplicity of notation. For any $\beta, \alpha>0$ and $0<m<1$ sufficiently small, let $(\hat{x}, \hat{t})$ be the maximum of $\Phi(x, t):=u(x, t)-v(x, t)-\beta\langle x\rangle^{m}-\alpha t$ in $\mathbb{R}^{n} \times[0, T]$, where $\langle x\rangle=$ $\left(1+|x|^{2}\right)^{1 / 2}$. In the rest of the proof we will suppose that $\beta+\alpha t_{0}<A$. Therefore $\Phi\left(x_{0}, t_{0}\right)>0$ and thus $\hat{t} \neq 0$. Moreover from $\Phi(\hat{x}, \hat{t}) \geqslant \Phi\left(x_{0}, t_{0}\right)$ we get

$$
u(\hat{x}, \hat{t})-v(\hat{x}, \hat{t})>2 \gamma>0 .
$$

In the nonstandard case we have $(\hat{x}, \hat{t}) \in \Gamma \times(0, T]$. Thus by the assumption, we suppose to fix ideas that we can find sequences $\varepsilon_{k} \rightarrow 0^{+}, \sigma_{\varepsilon_{k}} \rightarrow 0, p^{\varepsilon_{k}} \in \mathbb{R}^{n}$ with $\left|p^{\varepsilon_{k}}\right| \rightarrow 0^{+}$, and $\sigma_{\varepsilon_{k}} \leqslant 0$ if $\hat{t}=T$, such that $\lim _{k \rightarrow+\infty} v\left(\hat{x}+\varepsilon_{k} \eta^{-}+\varepsilon_{k} p^{\varepsilon_{k}}, \hat{t}+\sigma_{\varepsilon_{k}}\right)=v(\hat{x}, \hat{t})$. We drop the index $k$ from now on. 
Notice that as $\varepsilon \rightarrow 0^{+}$we can always find $p^{\varepsilon}$ with $\left|p^{\varepsilon}\right| \rightarrow 0$ and $\sigma_{\varepsilon} \rightarrow 0$ such that

$$
\lim _{\varepsilon \rightarrow 0^{+}} v\left(\hat{x}+\varepsilon\left(p^{\varepsilon}+\eta\right), \hat{t}+\sigma_{\varepsilon}\right)=v(\hat{x}, \hat{t}),
$$

where

$$
\begin{cases}\eta=0 & \text { if } \hat{x} \notin \Gamma \\ \eta=\eta^{-} & \text {if } \hat{x} \in \Gamma .\end{cases}
$$

We now define

$$
\begin{aligned}
\omega^{\varepsilon}(x, y, t, s):= & u(x, t)-v(y, s)-\frac{\gamma}{2}\left(\left|\frac{x-y}{\varepsilon}+\eta\right|^{2}+\left|\frac{t-s}{\sqrt{\left|\sigma_{\varepsilon}\right|}}\right|^{2}\right) \\
& -\frac{r}{2}\left(|x-\hat{x}|^{2}+|t-\hat{t}|^{2}\right)-\beta\langle x\rangle^{m}-\alpha t
\end{aligned}
$$

and consider $\left(x_{\varepsilon}, y_{\varepsilon}, t_{\varepsilon}, s_{\varepsilon}\right) \in \mathbb{R}^{2 n} \times[0, T]^{2}$ such that

$$
\omega^{\varepsilon}\left(x_{\varepsilon}, y_{\varepsilon}, t_{\varepsilon}, s_{\varepsilon}\right)=\max \left\{\omega^{\varepsilon}(x, y, t, s):(x, y, t, s) \in \mathbb{R}^{2 n} \times[0, T]^{2}\right\} .
$$

By definition $\omega^{\varepsilon}\left(x_{\varepsilon}, y_{\varepsilon}, t_{\varepsilon}, s_{\varepsilon}\right) \geqslant \omega^{\varepsilon}(\hat{x}, \hat{x}, \hat{t}, \hat{t})=\Phi(\hat{x}, \hat{t})-\frac{\gamma}{2}|\eta|^{2}>0$ for a sufficiently small $\gamma$. Hence the sequences $x_{\varepsilon}, y_{\varepsilon}$ are bounded and $\left|x_{\varepsilon}-y_{\varepsilon}\right| \leqslant(C+1) \varepsilon,\left|t_{\varepsilon}-s_{\varepsilon}\right| \leqslant C \sqrt{\left|\sigma_{\varepsilon}\right|}$, for some $C>0$. We therefore find that

$$
\lim _{\varepsilon \rightarrow 0^{+}}\left(x_{\varepsilon}, y_{\varepsilon}, t_{\varepsilon}, s_{\varepsilon}\right)=(\bar{x}, \bar{x}, \bar{t}, \bar{t}) \in \mathbb{R}^{2 n} \times[0, T]^{2} .
$$

By semicontinuity of $u, v$,

$$
\begin{aligned}
\Phi(\bar{x}, \bar{t}) & =u(\bar{x}, \bar{t})-v(\bar{x}, \bar{t})-\beta\langle\bar{x}\rangle^{m}-\alpha \bar{t} \\
& \geqslant \limsup _{\varepsilon \rightarrow 0^{+}}\left(u\left(x_{\varepsilon}, t_{\varepsilon}\right)-v\left(y_{\varepsilon}, s_{\varepsilon}\right)-\beta\left\langle x_{\varepsilon}\right\rangle^{m}-\alpha t_{\varepsilon}\right) \\
& \geqslant \liminf _{\varepsilon \rightarrow 0^{+}}\left(\omega^{\varepsilon}\left(x_{\varepsilon}, y_{\varepsilon}, t_{\varepsilon}, s_{\varepsilon}\right)+\frac{r}{2}\left(\left|x_{\varepsilon}-\hat{x}\right|^{2}+\left|t_{\varepsilon}-\hat{t}\right|^{2}\right)\right) \\
& \geqslant \liminf _{\varepsilon \rightarrow 0^{+}}\left(\omega^{\varepsilon}\left(\hat{x}, \hat{x}+\varepsilon\left(p^{\varepsilon}+\eta\right), \hat{t}, \hat{t}+\sigma_{\varepsilon}\right)+\frac{r}{2}\left(\left|x_{\varepsilon}-\hat{x}\right|^{2}+\left|t_{\varepsilon}-\hat{t}\right|^{2}\right)\right) \\
& =\liminf _{\varepsilon \rightarrow 0^{+}}\left(u(\hat{x}, \hat{t})-v\left(\hat{x}+\varepsilon\left(p^{\varepsilon}+\eta\right), \hat{t}+\sigma_{\varepsilon}\right)-\frac{\gamma}{2}\left(\left|p^{\varepsilon}\right|^{2}+\left|\sigma_{\varepsilon}\right|\right)\right. \\
& \left.\quad-\beta\langle\hat{x}\rangle^{m}-\alpha \hat{t}+\frac{r}{2}\left(\left|x_{\varepsilon}-\hat{x}\right|^{2}+\left|t_{\varepsilon}-\hat{t}\right|^{2}\right)\right) \\
& =\Phi(\hat{x}, \hat{t})+\frac{r}{2}\left(|\bar{x}-\hat{x}|^{2}+|\bar{t}-\hat{t}|^{2}\right) .
\end{aligned}
$$

Hence, as $(\hat{x}, \hat{t})$ is a maximum point of $\Phi$ in $\mathbb{R}^{n} \times[0, T]$, we obtain $\bar{x}=\hat{x}, \bar{t}=\hat{t}$ and

$$
\lim _{\varepsilon \rightarrow 0^{+}}\left(u\left(x_{\varepsilon}, t_{\varepsilon}\right)-v\left(y_{\varepsilon}, s_{\varepsilon}\right)\right)=u(\hat{x}, \hat{t})-v(\hat{x}, \hat{t}) .
$$

We make this information more precise by observing that

$$
\begin{aligned}
u(\hat{x}, \hat{t}) & \geqslant \limsup _{\varepsilon \rightarrow 0^{+}} u\left(x_{\varepsilon}, t_{\varepsilon}\right) \geqslant \liminf _{\varepsilon \rightarrow 0^{+}} u\left(x_{\varepsilon}, t_{\varepsilon}\right)=\liminf _{\varepsilon \rightarrow 0^{+}}\left(\left(u\left(x_{\varepsilon}, t_{\varepsilon}\right)-v\left(y_{\varepsilon}, s_{\varepsilon}\right)\right)+v\left(y_{\varepsilon}, s_{\varepsilon}\right)\right) \\
& \geqslant(u(\hat{x}, \hat{t})-v(\hat{x}, \hat{t}))+v(\hat{x}, \hat{t})=u(\hat{x}, \hat{t}),
\end{aligned}
$$


and so

Again from

$$
\lim _{\varepsilon \rightarrow 0^{+}} u\left(x_{\varepsilon}, t_{\varepsilon}\right)=u(\hat{x}, \hat{t}), \quad \lim _{\varepsilon \rightarrow 0^{+}} v\left(y_{\varepsilon}, s_{\varepsilon}\right)=v(\hat{x}, \hat{t}) .
$$

$\omega^{\varepsilon}\left(\hat{x}, \hat{x}+\varepsilon\left(p^{\varepsilon}+\eta\right), \hat{t}, \hat{t}+\sigma_{\varepsilon}\right) \leqslant \omega^{\varepsilon}\left(x_{\varepsilon}, y_{\varepsilon}, t_{\varepsilon}, s_{\varepsilon}\right) \leqslant u\left(x_{\varepsilon}, t_{\varepsilon}\right)-v\left(y_{\varepsilon}, s_{\varepsilon}\right)$

$$
-\frac{\gamma}{2}\left(\left|\frac{x_{\varepsilon}-y_{\varepsilon}}{\varepsilon}+\eta\right|^{2}+\left|\frac{t_{\varepsilon}-s_{\varepsilon}}{\sqrt{\left|\sigma_{\varepsilon}\right|}}\right|^{2}\right)-\beta\left\langle x_{\varepsilon}\right\rangle^{m}-\alpha t_{\varepsilon}
$$

we obtain

$$
\lim _{\varepsilon \rightarrow 0^{+}}\left|\frac{x_{\varepsilon}-y_{\varepsilon}}{\varepsilon}+\eta\right|=0, \quad \lim _{\varepsilon \rightarrow 0^{+}}\left|\frac{t_{\varepsilon}-s_{\varepsilon}}{\sqrt{\left|\sigma_{\varepsilon}\right|}}\right|=0 ;
$$

and hence for $\varepsilon$ sufficiently small,

$$
\left|x_{\varepsilon}-y_{\varepsilon}+\varepsilon \eta\right| \leqslant c \varepsilon,
$$

where $c>0$ appears in Remark 2.3 In particular if $\hat{x} \in \Gamma$ and $x_{\varepsilon} \in \Omega^{-} \cup \Gamma$, then $y_{\varepsilon} \in \Omega^{-}$, which is something that we keep in mind for later.

Since $t_{\varepsilon}, s_{\varepsilon} \in(0, T]$ we can use the definition of subsolution and supersolution (and Lemma 3.3) and compute, respectively,

$$
\begin{aligned}
0 \geqslant & \frac{\gamma}{\sqrt{\left|\sigma_{\varepsilon}\right|}}\left(\frac{t_{\varepsilon}-s_{\varepsilon}}{\sqrt{\left|\sigma_{\varepsilon}\right|}}\right)+r\left(t_{\varepsilon}-\hat{t}\right)+\alpha \\
& +f_{*}\left(x_{\varepsilon}\right) H\left(x_{\varepsilon}, \frac{\gamma}{\varepsilon}\left(\frac{x_{\varepsilon}-y_{\varepsilon}}{\varepsilon}+\eta\right)+r\left(x_{\varepsilon}-\hat{x}\right)+\beta m x_{\varepsilon}\left\langle x_{\varepsilon}\right\rangle^{m-2}\right),
\end{aligned}
$$

and

$$
0 \leqslant \frac{\gamma}{\sqrt{\left|\sigma_{\varepsilon}\right|}}\left(\frac{t_{\varepsilon}-s_{\varepsilon}}{\sqrt{\left|\sigma_{\varepsilon}\right|}}\right)+f^{*}\left(y_{\varepsilon}\right) H\left(y_{\varepsilon}, \frac{\gamma}{\varepsilon}\left(\frac{x_{\varepsilon}-y_{\varepsilon}}{\varepsilon}+\eta\right)\right) .
$$

Combining the two inequalities we obtain

$$
\begin{aligned}
r\left(t_{\varepsilon}-\hat{t}\right)+\alpha \leqslant & f^{*}\left(y_{\varepsilon}\right) H\left(y_{\varepsilon}, \frac{\gamma}{\varepsilon}\left(\frac{x_{\varepsilon}-y_{\varepsilon}}{\varepsilon}+\eta\right)\right) \\
& -f_{*}\left(x_{\varepsilon}\right) H\left(x_{\varepsilon}, \frac{\gamma}{\varepsilon}\left(\frac{x_{\varepsilon}-y_{\varepsilon}}{\varepsilon}+\eta\right)+r\left(x_{\varepsilon}-\hat{x}\right)+\beta m x_{\varepsilon}\left\langle x_{\varepsilon}\right\rangle^{m-2}\right) \\
\leqslant & {\left[f^{*}\left(y_{\varepsilon}\right)-f_{*}\left(x_{\varepsilon}\right)\right] H\left(y_{\varepsilon}, \frac{\gamma}{\varepsilon}\left(\frac{x_{\varepsilon}-y_{\varepsilon}}{\varepsilon}+\eta\right)\right)+\|f\|_{\infty} \mid H\left(y_{\varepsilon}, \frac{\gamma}{\varepsilon}\left(\frac{x_{\varepsilon}-y_{\varepsilon}}{\varepsilon}+\eta\right)\right) } \\
& -H\left(x_{\varepsilon}, \frac{\gamma}{\varepsilon}\left(\frac{x_{\varepsilon}-y_{\varepsilon}}{\varepsilon}+\eta\right)+r\left(x_{\varepsilon}-\hat{x}\right)+\beta m x_{\varepsilon}\left\langle x_{\varepsilon}\right\rangle^{m-2}\right) \mid .
\end{aligned}
$$

Now we analyze the two terms on the right hand side of 3.77 as $\varepsilon \rightarrow 0$. We start with the first term if $\hat{x} \notin \Gamma$, or else if $\hat{x} \in \Gamma$ and $x_{\varepsilon} \in \Omega^{-} \cup \Gamma$ thus $y_{\varepsilon} \in \Omega^{-}$, for all $\varepsilon$ sufficiently small. We get

$$
\begin{aligned}
{\left[f_{*}\left(y_{\varepsilon}\right)-f_{*}\left(x_{\varepsilon}\right)\right] H\left(y_{\varepsilon}, \frac{\gamma}{\varepsilon}\left(\frac{x_{\varepsilon}-y_{\varepsilon}}{\varepsilon}+\eta\right)\right) } & \leqslant L_{f}\left|x_{\varepsilon}-y_{\varepsilon}\right| H\left(y_{\varepsilon}, \frac{\gamma}{\varepsilon}\left(\frac{x_{\varepsilon}-y_{\varepsilon}}{\varepsilon}+\eta\right)\right) \\
& =L_{f} \gamma \frac{\left|x_{\varepsilon}-y_{\varepsilon}\right|}{\varepsilon} H\left(y_{\varepsilon},\left(\frac{x_{\varepsilon}-y_{\varepsilon}}{\varepsilon}+\eta\right)\right) \rightarrow 0
\end{aligned}
$$


by 3.5 and the homogeneity of $H$. If instead $\hat{x} \in \Gamma$ and $x_{\varepsilon} \in \Omega^{+}$along a subsequence, we have two further cases: either $y_{\varepsilon} \in \Omega^{+} \cup \Gamma$ for $\varepsilon$ small and we proceed again as above, or $y_{\varepsilon} \in \Omega^{-}$ along a subsequence. In the latter situation we have

$$
\begin{aligned}
\limsup _{\varepsilon \rightarrow 0^{+}}\left([ f ( y _ { \varepsilon } ) - f ( x _ { \varepsilon } ) ] H \left(y_{\varepsilon}\right.\right. & \left.\left.\frac{\gamma}{\varepsilon}\left(\frac{x_{\varepsilon}-y_{\varepsilon}}{\varepsilon}+\eta\right)\right)\right) \\
& \leqslant \underbrace{\left[f_{*}(\hat{x})-f^{*}(\hat{x})\right]}_{<0} \limsup _{\varepsilon \rightarrow 0^{+}} H\left(y_{\varepsilon}, \frac{\gamma}{\varepsilon}\left(\frac{x_{\varepsilon}-y_{\varepsilon}}{\varepsilon}+\eta\right)\right) \leqslant 0,
\end{aligned}
$$

since $H \geqslant 0$.

We now analyze the second term on the right hand side of (3.7). This is standard since the discontinuity of $f$ does not play any role. We get

$$
\begin{aligned}
\left|H\left(y_{\varepsilon}, \frac{\gamma}{\varepsilon}\left(\frac{x_{\varepsilon}-y_{\varepsilon}}{\varepsilon}+\eta\right)\right)-H\left(x_{\varepsilon}, \frac{\gamma}{\varepsilon}\left(\frac{x_{\varepsilon}-y_{\varepsilon}}{\varepsilon}+\eta\right)+r\left(x_{\varepsilon}-\hat{x}\right)+\beta m x_{\varepsilon}\left\langle x_{\varepsilon}\right\rangle^{m-2}\right)\right| \\
=\gamma\left|H\left(y_{\varepsilon}, \frac{1}{\varepsilon}\left(\frac{x_{\varepsilon}-y_{\varepsilon}}{\varepsilon}+\eta\right)\right)-H\left(x_{\varepsilon}, \frac{1}{\varepsilon}\left(\frac{x_{\varepsilon}-y_{\varepsilon}}{\varepsilon}+\eta\right)\right)\right| \\
\quad+\mid H\left(x_{\varepsilon}, \frac{\gamma}{\varepsilon}\left(\frac{x_{\varepsilon}-y_{\varepsilon}}{\varepsilon}+\eta\right)\right)-H\left(x_{\varepsilon}, \frac{\gamma}{\varepsilon}\left(\frac{x_{\varepsilon}-y_{\varepsilon}}{\varepsilon}+\eta\right)+r\left(x_{\varepsilon}-\hat{x}\right)+\beta m x_{\varepsilon}\left\langle\left. x_{\varepsilon}\right|^{m-2}\right) \mid\right. \\
\leqslant \gamma \omega_{\hat{x}}\left(\left|x_{\varepsilon}-y_{\varepsilon}\right|+\frac{\left|x_{\varepsilon}-y_{\varepsilon}+\varepsilon \eta\right|^{2}}{\varepsilon^{2}}\right)+\omega_{K}\left(\left|r\left(x_{\varepsilon}-\hat{x}\right)+\beta m x_{\varepsilon}\left\langle x_{\varepsilon}\right\rangle^{m-2}\right|\right) \\
\leqslant \gamma \omega_{\hat{x}}\left(\left|x_{\varepsilon}-y_{\varepsilon}\right|+\frac{\left|x_{\varepsilon}-y_{\varepsilon}+\varepsilon \eta\right|^{2}}{\varepsilon^{2}}\right)+\omega_{K}\left(r\left|x_{\varepsilon}-\hat{x}\right|+\beta m\right) \rightarrow \omega_{K}(\beta m) \quad \text { as } \varepsilon \rightarrow 0^{+},
\end{aligned}
$$

by 2.1 -2.3, where $K=B(\hat{x}, r)$.

By taking the lim sup in (3.7) we finally obtain

$$
\alpha \leqslant 0+\|f\|_{\infty} \omega_{K}(\beta m),
$$

hence a contradiction for $m$ sufficiently small and given $\alpha, \beta$.

The following uniqueness result is an immediate consequence.

Corollary 3.5 Assume 2.1]-2.3 and 1.2 . A viscosity solution $u \in C\left(\mathbb{R}^{n} \times[0,+\infty)\right)$ of (1.1) is unique within the class of discontinuous solutions.

The scope of the comparison principle can be extended to equations having an explicit $t$ dependence. The assumptions become slightly more technical and restrictive, and this case will not be used explicitly in the next section. However the proofs are quite similar and the results have independent interest so we give here exact assumptions and statements without proofs. In the rest of the section we consider the following problem $(T>0)$ :

$$
\left\{\begin{array}{l}
u_{t}(x, t)+f(x, t) \tilde{H}(x, t, D u(x, t))=0, \quad(x, t) \in \mathbb{R}^{n} \times(0, T), \\
u(x, 0)=u_{0}(x) \in C\left(\mathbb{R}^{n}\right) .
\end{array}\right.
$$

The discontinuity set of the coefficient $f$ is now $\tilde{\Gamma} \subset \mathbb{R}^{n} \times[0,+\infty)$ and $f$ will be piecewise Lipschitz across a finite union of disjoint Lipschitz hypersurfaces. We also replace the assumptions 
2.2), 2.3 with the following: for any $(\hat{x}, \hat{t}) \in \mathbb{R}^{n} \times(0,+\infty)$ and $\rho>0$ sufficiently small,

$$
\begin{aligned}
\tilde{H}\left(x, t, \alpha(x-y)+\sqrt{\alpha} \eta_{x}\right)-\tilde{H}(y, s, \alpha(x-y) & \left.+\sqrt{\alpha} \eta_{x}\right) \\
& \geqslant-\omega_{\hat{x}, \hat{t}}\left(|x-y|+\alpha\left|x-y+\frac{1}{\sqrt{\alpha}} \eta_{x}\right|^{2}\right)
\end{aligned}
$$

for all $(x, t),(y, s) \in B((\hat{x}, \hat{t}), \rho), \alpha>0$, with $\eta=\left(\eta_{x}, \eta_{t}\right)=\eta^{-}$a unit vector pointing into $\Omega^{-}$if $(\hat{x}, \hat{t}) \in \tilde{\Gamma}$ and $\eta=0$ if $(\hat{x}, \hat{t}) \in\left(\mathbb{R}^{n} \times(0,+\infty)\right) \backslash \tilde{\Gamma}$

$$
\begin{aligned}
\tilde{H}\left(x, t, \alpha(x-y)-\sqrt{\alpha} \eta_{x}\right)-\tilde{H}\left(y, s, \alpha(x-y)-\sqrt{\alpha} \eta_{x}\right) & \\
& \geqslant-\omega_{\hat{x}, \hat{t}}\left(|x-y|+\alpha\left|x-y-\frac{1}{\sqrt{\alpha}} \eta_{x}\right|^{2}\right)
\end{aligned}
$$

for all $(x, t),(y, s) \in B((\hat{x}, \hat{t}), \rho), \alpha>0$, with $\eta=\eta^{+}$a unit vector pointing into $\Omega^{+}$if $(\hat{x}, \hat{t}) \in \tilde{\Gamma}$ and $\eta=0$ if $(\hat{x}, \hat{t}) \in\left(\mathbb{R}^{n} \times(0,+\infty)\right) \backslash \tilde{\Gamma}$.

Then we can prove the following result. Below, the assumptions 1.2 and 2.1 are obviously adapted to the presence of the new variable $t$.

TheOREM 3.6 Let $T \in(0,+\infty]$ and assume (2.1), (3.9), (3.10) and (1.2). Let $u, v: \mathbb{R}^{n} \times[0, T]$ $\rightarrow \mathbb{R}$ be respectively an upper semicontinuous subsolution and a lower semicontinuous supersolution of the HJ equation

$$
w_{t}(x, t)+f(x, t) H(x, t, D w(x, t))=0, \quad(x, t) \in \mathbb{R}^{n} \times(0, T),
$$

such that

$$
u(x, T)=\limsup _{(y, s) \rightarrow\left(x, T^{-}\right)} u(y, s), \quad v(x, T)=\liminf _{(y, s) \rightarrow\left(x, T^{-}\right)} v(y, s) .
$$

Assume moreover that $u(x, 0) \leqslant v(x, 0)$ for $x \in \mathbb{R}^{n}$ and that for all $(\hat{x}, \hat{t}) \in \Gamma \times(0, T], \hat{t}>0$, we can find sequences $\varepsilon_{k} \rightarrow 0^{+}$and $p^{\varepsilon_{k}} \in \mathbb{R}^{n+1}$ with $\left|p^{\varepsilon_{k}}\right| \rightarrow 0^{+}$such that either

$$
\lim _{k \rightarrow+\infty} u\left((\hat{x}, \hat{t})+\varepsilon_{k} \eta^{+}+\varepsilon_{k} p^{\varepsilon_{k}}\right)=u(\hat{x}, \hat{t})
$$

or

$$
\lim _{k \rightarrow+\infty} v\left((\hat{x}, \hat{t})+\varepsilon_{k} \eta^{-}+\varepsilon_{k} p^{\varepsilon_{k}}\right)=v(\hat{x}, \hat{t}),
$$

where $\eta^{ \pm}$are unit vectors pointing into $\Omega^{ \pm}$on $\left(\mathbb{R}^{n} \times(0, T]\right) \cap \tilde{\Gamma}$. Then $u \leqslant v$ in $\mathbb{R}^{n} \times[0, T]$.

We record the above result for convenience, since its proof is a minor modification of that of Theorem 3.1, but the existence part in the next section will not use it. Our approach to existence will be limited to Hamiltonians independent of $t$.

\section{Existence of continuous viscosity solutions}

In order to use the control-theoretic interpretation of solutions and avoid dealing with discontinuous vector fields, we rather look at $(1.1)$ as the following Bellman-Isaacs equation:

$$
\begin{cases}\frac{u_{t}(x, t)}{f(x)}+H(x, D u(x, t))=0, & (x, t) \in \mathbb{R}^{n} \times(0,+\infty), \\ u(x, 0)=u_{0}(x), & x \in \mathbb{R}^{n},\end{cases}
$$


where $H$ has the structure (1.3), and construct the corresponding value function. Notice that the Hamiltonian does not change if we substitute the control set $A$ with the relaxed controls $P(A)$, the Radon probability measures on $A$. In this case, for any $\mu \in P(A)$, we extend $g$ as follows:

$$
g(x, \mu, b)=\int_{A} g(x, a, b) \mathrm{d} \mu(a)
$$

and identify $a \in A$ with the Dirac mass $\delta_{a} \in P(A)$. This is a technical point, since, roughly, relaxation implies existence of minima in integral functionals under appropriate assumptions. The reader can consult Warga [24] for relaxed controls in optimal control, Elliott-Kalton [11] for the case of differential games and also [1].

Thus we consider the following control system:

$$
\begin{cases}\dot{x}(s)=g(x(s), a(s), b(s)), & x(0)=x_{0}, \\ “ t(s)=t_{0}-\int_{0}^{s} \frac{1}{f(x(r))} \mathrm{d} r, & t_{0}>0,\end{cases}
$$

where we will make precise the second equation by using the semicontinuous envelopes of $f$. This makes a difference on the jump set. From now on $x(\cdot)=x\left(\cdot ; x_{0}, a(\cdot), b(\cdot)\right)$ will be a trajectory of the first equation in (4.2) when the two players choose the relaxed control functions $a(\cdot) \in$ $L^{\infty}(0,+\infty ; P(A))$ and $b(\cdot) \in L^{\infty}(0,+\infty ; B)$ respectively. The first set is metrizable and compact in the weak* topology.

We now introduce the set of nonanticipating or causal strategies for the controller $a$,

$$
\Delta=\left\{\alpha: L^{\infty}(0,+\infty ; B) \rightarrow L^{\infty}(0,+\infty ; P(A)): \alpha \text { causal }\right\}
$$

where $\alpha$ causal means that $b_{1}(t)=b_{2}(t)$ a.e. in $[0, T]$ for some $T>0$ implies $\alpha\left[b_{1}\right](t)=\alpha\left[b_{2}\right](t)$ a.e. in $[0, T]$. The notion of causality is intriguing; it has been introduced in this form by Varaya, Roxin, Elliott and Kalton (see [11] and the references therein for the history of this and other notions), and roughly means that the player $a$ at time $T$ only knows its opponent's choices up to time $T$. Causality is the key idea needed to extend to differential games Bellman's Dynamic Programming Principle and the connection of value functions to Hamilton-Jacobi equations (see also [1]). We have two possible candidate value functions. If we set $\hat{t}(s)=t_{0}-\int_{0}^{s}\left(1 / f^{*}(x(s))\right) \mathrm{d} s$, and $\hat{\tau}_{x_{0}, t_{0}}$ is such that $\hat{t}\left(\hat{\tau}_{x_{0}, t_{0}}\right)=0$, then we define

$$
\hat{v}\left(x_{0}, t_{0}\right)=\inf _{\alpha \in \Delta} \sup _{b \in L^{\infty}(0,+\infty ; B)} u_{0}\left(x\left(\hat{\tau}_{x_{0}, t_{0}} ; x_{0}, \alpha[b], b\right)\right) .
$$

If on the other hand $\check{t}(s)=t_{0}-\int_{0}^{s}\left(1 / f_{*}(x(s))\right) \mathrm{d} s$, and $\check{\tau}_{x_{0}, t_{0}}$ is such that $\check{t}\left(\check{\tau}_{x_{0}, t_{0}}\right)=0$, then we define

$$
\check{v}\left(x_{0}, t_{0}\right)=\inf _{\alpha \in \Delta} \sup _{b \in L^{\infty}(0,+\infty ; B)} u_{0}\left(x\left(\check{\tau}_{x_{0}, t_{0}} ; x_{0}, \alpha[b], b\right)\right) .
$$

We claim that under suitable assumptions $\hat{v}$ and $\breve{v}$ coincide and are the unique continuous solution of the Cauchy problem (1.1) (and (4.1)).

REMARK 4.1 We derive a consequence from the assumption $H \geqslant 0$. We notice that for all $\hat{x} \in \mathbb{R}^{n}$ we can find a strategy $\alpha_{\hat{x}} \in \Delta$ such that $x\left(t ; \hat{x}, \alpha_{\hat{x}}[b], b\right) \equiv \hat{x}$ for all $t \geqslant 0$ and $b \in L^{\infty}(0,+\infty ; B)$. A consequence of this fact is that any value function (say $\hat{v}$ for instance) satisfies $\hat{v}(x, t) \leqslant u_{0}(x)$ for 
$t \geqslant 0$ and thus by the dynamic programming principle $\hat{v}(x, \cdot)$ is nonincreasing. Indeed, the function $u(x)=|x-\hat{x}|^{2}$ is a viscosity supersolution of $H(x, D u(x)) \geqslant 0$. Then by the representation formula in Proposition 4.1 of [19] we have

$$
u(x)=\inf _{\alpha \in \Delta} \sup _{b \in L^{\infty}(0,+\infty ; B)} \sup _{t \geqslant 0} u(x(t)) .
$$

Therefore, by choosing at $\hat{x}$ an optimal strategy $\alpha_{\hat{x}}$ in 4.3 , which exists by the compactness of $\Delta$ and the continuity of $u$ (see [11]), we have

$$
0=u(\hat{x}) \geqslant|x(t)-\hat{x}|^{2}
$$

for all $b(\cdot)$ and $t \geqslant 0$. The proof of $(4.3)$ is somewhat technical, but its consequence can be well understood in the case that $B$ is a singleton. If

$$
H(x, p)=\max _{a \in A}\{-g(x, a) \cdot p\} \geqslant 0,
$$

then for all $p \in \mathbb{R}^{n}$ we can find $a \in A$ such that $g(x, a) \cdot p \leqslant 0$. Thus

$$
0 \in g(x, P(A))=\overline{\operatorname{co}} g(x, A) \quad \text { for all } x \in \mathbb{R}^{n},
$$

where the notation $\overline{\mathrm{co}}$ indicates the closed, convex hull. We can therefore find $a_{x} \in P(A)$ such that $g\left(x, a_{x}\right)=0$ and $x\left(t ; \hat{x}, a_{x}\right) \equiv x$.

REMARK 4.2 Suppose that $f_{1}(x) \geqslant f_{2}(x) \geqslant c_{0}>0$. Then for $(x, t) \in \mathbb{R}^{n} \times(0,+\infty)$, all strategies $\alpha \in \Delta$ and all controls $b(\cdot) \in L^{\infty}(0,+\infty ; B)$ we define $\tau^{i}=\tau^{i}(\alpha[b], b), i=1,2$, by setting

$$
t=\int_{0}^{\tau^{i}} \frac{1}{f_{i}(x(s))} \mathrm{d} s .
$$

Each $\tau^{i}$ is well defined since $f$ is strictly positive. We have $\tau^{1} \geqslant \tau^{2}$ since

$$
\int_{0}^{\tau^{1}} \frac{1}{f_{2}(x(s))} \mathrm{d} s \geqslant \int_{0}^{\tau^{1}} \frac{1}{f_{1}(x(s))} \mathrm{d} s=\int_{0}^{\tau^{2}} \frac{1}{f_{2}(x(s))} \mathrm{d} s .
$$

Now for given $x \in \mathbb{R}^{n}$ we modify the strategy by setting

$$
\tilde{\alpha}[b](s):= \begin{cases}\alpha[b](s), & s \leqslant \tau^{2}, \\ \alpha_{x\left(\tau^{2} ; x, \alpha[b], b\right)}\left[b\left(\cdot+\tau^{2}\right)\right]\left(s-\tau^{2}\right), & s>\tau^{2},\end{cases}
$$

for all $b \in L^{\infty}(0,+\infty ; B)$. We obtain

$$
x\left(\tau^{2}(\alpha[b], b) ; x, \alpha[b], b\right)=x\left(\tau^{2}(\tilde{\alpha}[b], b) ; x, \tilde{\alpha}[b], b\right)=x\left(\tau^{1}(\tilde{\alpha}[b], b) ; x, \tilde{\alpha}[b], b\right)
$$

for all $b \in L^{\infty}(0,+\infty ; B)$. Hence the corresponding value functions satisfy the relationship

$$
u_{1}(x, t)=\inf _{\alpha \in \Delta} \sup _{b \in L^{\infty}(0,+\infty ; B)} u_{0}\left(x\left(\tau^{1}\right)\right) \leqslant \inf _{\alpha \in \Delta} \sup _{b \in L^{\infty}(0,+\infty ; B)} u_{0}\left(x\left(\tau^{2}\right)\right)=u_{2}(x, t) .
$$

A first consequence of Remark 4.2 is that $\hat{v} \leqslant \check{v}$. 
We show that the Isaacs equation is satisfied by approximation with problems without discontinuities. We prove the following general result.

THEOREM 4.3 Suppose that the Hamiltonian is given by (1.3), and assume (2.4) and (1.2).

(i) The function $\hat{v}$ is lower semicontinuous in $\mathbb{R}^{n} \times[0,+\infty)$, and continuous at the points of $\left\{(x, 0): x \in \mathbb{R}^{n}\right\}$.

(ii) If we approximate $f^{*}$ (from above) by the family of Lipschitz continuous functions

$$
f^{\varepsilon}(x)=\sup _{y}\left\{f^{*}(y)-\frac{|x-y|^{2}}{2 \varepsilon^{2}}\right\},
$$

then $\hat{v}(x, t)=\sup _{\mathcal{E} \downarrow 0} v^{\varepsilon}(x, t)$, where $v^{\varepsilon} \in C\left(\mathbb{R}^{n} \times[0,+\infty)\right)$ solves the HJ equation in 1.1 , with $f$ replaced by $f^{\varepsilon}$.

(iii) $\hat{v}$ is the minimal viscosity solution of 1.1 .

Proof. We start by observing that the sequence $f^{\varepsilon}$ has uniform bounds, since $c_{0} \leqslant f^{*} \leqslant f^{\mathcal{E}} \leqslant$ $\|f\|_{\infty}$. Moreover by well known results, each sup-convolution $f^{\varepsilon}$ is Lipschitz continuous, $f^{\varepsilon} \downarrow f^{*}$, and thus

$$
\begin{aligned}
f^{*}(x) & =\inf _{\varepsilon} f^{\varepsilon}(x)=\lim _{r \rightarrow 0^{+}} \sup _{|y-x|<r, 0<\varepsilon<r} f^{\varepsilon}(y)\left(=: \limsup _{\varepsilon \rightarrow 0^{+}} f^{\varepsilon}(x)\right), \\
f_{*}(x) & =\lim _{r \rightarrow 0^{+}} \inf _{|y-x|<r, 0<\varepsilon<r} f^{\varepsilon}(y)\left(=: \liminf _{\varepsilon \rightarrow 0^{+}} f^{\varepsilon}(x)\right) .
\end{aligned}
$$

We consider the approximating Cauchy problem

$$
\left\{\begin{array}{l}
u_{t}(x, t)+f^{\varepsilon}(x) H(x, D u(x, t))=0 \\
u(x, 0)=u_{0}(x)
\end{array}\right.
$$

and for any given $\left(x_{0}, t_{0}\right) \in \mathbb{R}^{n} \times[0,+\infty)$ and controls $a(\cdot), b(\cdot)$, define the unique $\hat{\tau}_{x_{0}, t_{0}}^{\varepsilon} \geqslant 0$ such that $0=t_{0}-\int_{0}^{\hat{\tau}_{x_{0}, t_{0}}^{\varepsilon}}\left(1 / f^{\varepsilon}(x(s))\right) \mathrm{d} s$, where $x(\cdot)=x\left(\cdot ; x_{0}, a, b\right)$. Notice that $t_{0} c_{0} \leqslant \hat{\tau}_{x_{0}, t_{0}}^{\varepsilon} \leqslant$ $t_{0}\|f\|_{\infty}$. In particular by the boundedness of $g$ every value function

$$
v^{\varepsilon}(x, t)=\inf _{\alpha \in \Delta} \sup _{b \in L^{\infty}(0,+\infty ; B)} u_{0}\left(x\left(\hat{\tau}_{x, t}^{\varepsilon}\right)\right)
$$

is continuous at the points of $\left\{(x, 0): x \in \mathbb{R}^{n}\right\}$. Indeed, this follows from

$$
\left|u_{0}\left(x\left(\hat{\tau}_{x_{0}, t_{0}}^{\varepsilon}\right)\right)-u_{0}(x)\right| \leqslant \omega_{x}\left(\left|x\left(\hat{\tau}_{x_{0}, t_{0}}^{\varepsilon}\right)-x_{0}\right|+\left|x-x_{0}\right|\right) \leqslant \omega_{x}\left(\|f\|_{\infty}\|g\|_{\infty} t_{0}+\left|x-x_{0}\right|\right),
$$

where $\omega_{x}$ is a local modulus of continuity for $u_{0}$. Thus

$$
\left|v^{\varepsilon}\left(x_{0}, t_{0}\right)-u_{0}(x)\right| \leqslant \omega_{x}\left(\|f\|_{\infty}\|g\|_{\infty} t_{0}+\left|x-x_{0}\right|\right) .
$$

We pause to observe that for the same reason this fact also holds for $\hat{v}$ and $\check{v}$. By classical results $v^{\varepsilon}$ is therefore the unique continuous viscosity solution of (4.6) (see e.g. [1]).

Observe now that, by Remark 4.2 , the family $\left\{v^{\varepsilon}\right\}$ increases as $\varepsilon \rightarrow 0^{+}$. Therefore we can define the lower semicontinuous function

$$
\underline{v}(x, t)=\sup _{\varepsilon} v^{\varepsilon}(x, t)=\liminf _{\varepsilon \rightarrow 0^{+}} v^{\varepsilon}(x, t),
$$


which also satisfies

$$
\underline{v}^{*}(x, t)=\limsup _{\varepsilon \rightarrow 0^{+}}^{*} v^{\varepsilon}(x, t) .
$$

By stability of viscosity solutions, it is then well known that $\underline{v}$ is a viscosity solution of the HJ equation in (1.1) (see [1]).

We now show that $\hat{v}=\underline{v}$. By Remark 4.2, it is clear that $v^{\varepsilon} \leqslant \hat{v}$ and then $\underline{v} \leqslant \hat{v}$. Now we suppose towards a contradiction that $\underline{v}(x, t)+2 \delta \leqslant \hat{v}(x, t)$ for some $(x, t) \in \mathbb{R}^{n} \times(0,+\infty)$ and $\delta>0$. By definition, for all $\varepsilon>0$ sufficiently small, we can choose a strategy $\alpha_{\varepsilon}$ such that

$$
u_{0}\left(x\left(\hat{\tau}_{x, t}^{\varepsilon} ; x, \alpha_{\varepsilon}[b], b\right)\right)+\delta \leqslant \hat{v}(x, t)
$$

for all $b \in L^{\infty}(0,+\infty ; B)$. We will find a strategy $\alpha^{\#} \in \Delta$ such that, for all $b \in L^{\infty}(0,+\infty ; B)$, at least for a subsequence,

$$
\lim _{\varepsilon \rightarrow 0} x\left(\hat{\tau}_{x, t}^{\varepsilon} ; x, \alpha_{\varepsilon}[b], b\right)=x\left(\hat{\tau}_{x, t} ; x, \alpha^{\#}[b], b\right) .
$$

This will contradict 4.7) by continuity of $u_{0}$ and definition of $\hat{v}$.

Consider the subsequence $\varepsilon_{n}=1 / n$. By viewing $\alpha_{1 / n}$ as an element of the product space

$$
L^{\infty}\left(0, t\|f\|_{\infty} ; P(A)\right)^{L^{\infty}\left(0, t\|f\|_{\infty} ; B\right)},
$$

which is compact in the product topology, we can find a subnet $\left\{\alpha_{\lambda}\right\}_{\lambda \in \Lambda}$ of $\left\{\alpha_{1 / n}\right\}$ and an element $\tilde{\alpha}$ of the product space such that

$$
\alpha_{\lambda}[b] \stackrel{*}{\rightarrow} \tilde{\alpha}[b]
$$

for all $b \in L^{\infty}\left(0, t\|f\|_{\infty} ; B\right)$. The functional $\tilde{\alpha}$ is a strategy because $\Delta$ is a closed subset of $L^{\infty}\left(0, t\|f\|_{\infty} ; P(A)\right)^{L^{\infty}\left(0, t\left\|_{f}\right\|_{\infty} ; B\right)}$ in the product topology, as is easily verified.

Since $L^{\infty}\left(0, t\|f\|_{\infty} ; P(A)\right)$ is metrizable, we can find a subsequence $\left\{n_{k}\right\}$, possibly depending on $b(\cdot)$ such that

$$
\alpha_{\varepsilon_{n_{k}}}[b] \stackrel{*}{\rightarrow} \tilde{\alpha}[b]
$$

Fix any $b \in L^{\infty}(0,+\infty ; B)$. From the properties of weak convergence and Lemma 4.4 that we postpone after the end of the proof, we know that

$$
\begin{gathered}
x\left(s ; x, \alpha_{\varepsilon_{n_{k}}}[b], b\right) \rightarrow x(s ; x, \tilde{\alpha}[b], b) \quad \text { for all } s \in[0,+\infty), \\
\hat{\tau}_{x, t}(\tilde{\alpha}[b], b) \geqslant \tilde{T}:=\limsup _{\varepsilon_{n_{k}} \rightarrow 0^{+}} \hat{\tau}_{x, t}^{\varepsilon_{n_{k}}}\left(\alpha_{\varepsilon_{n_{k}}}[b], b\right) .
\end{gathered}
$$

We then restrict ourselves to a further subsequence that we now simply denote $\alpha_{\varepsilon_{n}}$ such that $\hat{\tau}_{x, t}^{\varepsilon_{n}}\left(\alpha_{\varepsilon_{n}}[b], b\right) \rightarrow \tilde{T}$ and get

$$
\begin{aligned}
\hat{x}\left(\hat{\tau}_{x, t}^{\varepsilon_{n}} ; x, \alpha_{\varepsilon_{n}}[b], b\right)= & x+\int_{0}^{\hat{\tau}_{x, t}^{\varepsilon_{n}}} g\left(x\left(s ; x, \alpha_{\varepsilon_{n}}[b], b\right), \alpha_{\varepsilon_{n}}[b], b\right) \mathrm{d} s \\
= & x+\int_{0}^{\tilde{T}} g\left(x\left(s ; x, \alpha_{\varepsilon_{n}}[b], b\right), \alpha_{\varepsilon_{n}}[b], b\right) \mathrm{d} s \\
& +\int_{\tilde{T}}^{\hat{\tau}_{x, t}^{\varepsilon_{n}}} g\left(x\left(s ; x, \alpha_{\varepsilon_{n}}[b], b\right), \alpha_{\varepsilon_{n}}[b], b\right) \mathrm{d} s \\
\rightarrow & x+\int_{0}^{\tilde{T}} g(x(s ; x, \tilde{\alpha}[b], b), \tilde{\alpha}[b], b)=x(\tilde{T} ; x, \tilde{\alpha}[b], b), \quad n \rightarrow \infty .
\end{aligned}
$$


We now modify the strategy $\tilde{\alpha}$ by setting

$$
\alpha^{\#}[b](s):= \begin{cases}\tilde{\alpha}[b](s), & s \leqslant \tilde{T}, \\ \alpha_{x(\tilde{T} ; x, \tilde{\alpha}[b], b)}[b(\cdot+\tilde{T})](s-\tilde{T}), & s>\tilde{T},\end{cases}
$$

and we obtain (4.8) as desired.

Concerning the fact that $\hat{v}$ is the minimal viscosity solution, observe that since $f^{\varepsilon} \geqslant f^{*} \geqslant f_{*}$, it follows that $v^{\varepsilon}$ is a continuous viscosity subsolution of 1.1. Thus by the comparison principle of the previous section, for any viscosity solution $v$ of 11.1 we have $v^{\varepsilon} \leqslant v_{*}$ and therefore $\hat{v} \leqslant v_{*}$.

It remains to show the claimed lemma.

Lemma $4.4 \hat{\tau}_{x, t}(\tilde{\alpha}[b], b) \geqslant \tilde{T}:=\lim \sup _{\varepsilon_{n_{k}} \rightarrow 0^{+}} \hat{\tau}_{x, t}^{\varepsilon_{n_{k}}}\left(\alpha_{\varepsilon_{n_{k}}}[b], b\right)$.

Proof. We restrict ourselves to a subsequence, that for simplicity we indicate with $\varepsilon_{n}$, such that $\tilde{T}:=\lim _{\varepsilon_{n} \rightarrow 0^{+}} \hat{\tau}_{x, t}^{\varepsilon_{n}}\left(\alpha_{\varepsilon_{n}}[b], b\right)$. By definition

$$
\int_{0}^{\hat{\tau}_{x, t}(\tilde{\alpha}[b], b)} \frac{1}{f^{*}(x(s ; x, \tilde{\alpha}[b], b))} \mathrm{d} s=t=\int_{0}^{\hat{\tau}_{x, t}^{\varepsilon_{n}}\left(\alpha_{\varepsilon_{n}}[b], b\right)} \frac{1}{f^{\varepsilon_{n}}\left(x\left(s ; x, \alpha_{\varepsilon_{n}}[b], b\right)\right)} \mathrm{d} s,
$$

and hence from Fatou's Lemma and the approximating properties of $f^{\varepsilon}$ in 4.5 we have

$$
t \geqslant \int_{0}^{\tilde{T}} \frac{1}{f^{*}(x(s ; x, \tilde{\alpha}[b], b))} \mathrm{d} s .
$$

The conclusion then follows.

The following is the corresponding statement for the approximation of the solution of our problem from above and has an identical proof.

THEOREM 4.5 Suppose that the Hamiltonian is given by (1.3), and assume (2.4) and (1.2). Given the inf-convolutions

$$
f_{\varepsilon}(x)=\inf _{y}\left\{f_{*}(y)+\frac{|x-y|^{2}}{2 \varepsilon^{2}}\right\},
$$

let $\bar{v}(x, t)=\inf _{\varepsilon \downarrow} v_{\varepsilon}(x, t)$, where $t=\int_{0}^{\tau_{x, t}^{\varepsilon}(\tilde{\alpha}[b], b)}\left(1 / f_{\varepsilon}(x(s ; x, \alpha[b], b))\right) \mathrm{d} s$ and

$$
v_{\varepsilon}(x, t)=\inf _{\alpha \in \Delta} \sup _{b \in L^{\infty}(0,+\infty ; B)} u_{0}\left(x\left(\check{\tau}_{x, t}^{\varepsilon}(\tilde{\alpha}[b], b)\right)\right),
$$

and $v_{\varepsilon} \in C\left(\mathbb{R}^{n} \times[0,+\infty)\right)$ solves the HJ equation in 1.1$]$ with $f$ replaced by $f_{\varepsilon}$. Then $\check{v} \leqslant \bar{v}$ and $\bar{v}$ is upper semicontinuous in $\mathbb{R}^{n} \times[0,+\infty)$, continuous at the points of $\left\{(x, 0): x \in \mathbb{R}^{n}\right\}$, and the maximal viscosity solution of 1.1 .

REMARK 4.6 By the classical dynamic programming principle we could show directly that $\check{v}$ is a viscosity solution of 1.1. This is a matter that we skip. We could not prove directly however that $\bar{v}=\check{v}$; our conjecture is that $\bar{v}=\breve{v}^{*}$, except in the case that $B$ is a singleton. So we claim that

$$
\check{v}(x, t)=\inf _{a \in L^{\infty}(0,+\infty ; P(A))} u_{0}\left(x\left(\check{\tau}_{x, t} ; x, a\right)\right),
$$

as we now show. 
Suppose on the contrary that for some $\delta>0$ and $(x, t) \in \mathbb{R}^{n} \times(0,+\infty)$,

$$
\bar{v}(x, t) \geqslant \check{v}(x, t)+2 \delta .
$$

Then we can find $\check{a} \in L^{\infty}(0,+\infty ; P(A))$ such that

$$
u_{0}\left(x\left(\check{\tau}_{x, t} ; x, \check{a}\right)\right)+\delta \leqslant \bar{v}(x, t) .
$$

If we verify that

$$
\lim _{\varepsilon \rightarrow 0^{+}} \check{\tau}_{x, t}^{\varepsilon}(\check{a})=\check{\tau}_{x, t}(\check{a})
$$

where

$$
\int_{0}^{\check{\tau}_{x, t}^{\varepsilon}(\check{a})} \frac{1}{f_{\varepsilon}(x(s ; x, \check{a}))} \mathrm{d} s=t,
$$

then we reach a contradiction to 4.9 for $\varepsilon$ sufficiently small, because

$$
\bar{v}(x, t) \leqslant v_{\varepsilon}(x, t) \leqslant u_{0}\left(x\left(\check{\tau}_{x, t}^{\varepsilon} ; x, \check{a}\right)\right) .
$$

Now we prove 4.10 . Since $\check{\tau}_{x, t}^{\varepsilon}$ is bounded, we take any converging subsequence $\check{\tau}_{x, t}^{\varepsilon_{n}}(\check{a}) \rightarrow \tilde{T}$. Then we pass to the limit in

$$
\int_{0}^{\check{\tau}_{x, t}(\check{a})} \frac{1}{f_{*}(x(s ; x, \check{a}))} \mathrm{d} s=t=\int_{0}^{\check{\tau}_{x, t}^{\varepsilon_{n}}(\check{a})} \frac{1}{f_{\varepsilon_{n}}(x(s ; x, \check{a}))} \mathrm{d} s
$$

and obtain

$$
\int_{0}^{\check{\tau}_{x, t}(\check{a})} \frac{1}{f_{*}(x(s ; x, \check{a}))} \mathrm{d} s=\int_{0}^{\tilde{T}} \frac{1}{f_{*}(x(s ; x, \check{a}))} \mathrm{d} s,
$$

hence $\check{\tau}_{x, t}(\check{a})=\tilde{T}$.

We have reached the following point.

COROLlary 4.7 Suppose that the Hamiltonian is given by (1.3), and assume (2.4) and (1.2). Then

$$
\hat{v} \leqslant \check{v} \leqslant \bar{v}
$$

and the last inequality is an equality in the case of a single player. The functions at the extremes are lower and upper semicontinuous, respectively, and viscosity solutions of (1.1).

REMARK 4.8 It is clear that if we interpret uniqueness of $(1.1)$ by requiring that $\hat{v}=\bar{v}$, then the unique solution must be continuous. This would rule out uniqueness when the viscosity solution is discontinuous, as in Example 2.1. However, we could also interpret uniqueness as the following requirements: $\hat{v}=(\bar{v})_{*}$ and $(\hat{v})^{*}=\bar{v}$. As a necessary condition this requires that $\left((\bar{v})_{*}\right)^{*}=\bar{v}$, $\left((\hat{v})^{*}\right)_{*}=\hat{v}$ and therefore conditions on the possible discontinuities of the solutions. We will not deal with this part of the problem. For some analysis of necessary conditions on the discontinuities of the viscosity solution in order to have uniqueness, we refer instead to [20, 22]. 
We wish now to prove that indeed under appropriate assumptions $\bar{v} \leqslant \hat{v}$ by using the comparison principle of Section 3, so there is a unique continuous solution of (1.1) which is the uniform limit of suitable approximations of continuous problems. Therefore we now discuss how to obtain the extra continuity properties of a value function that we need in order to apply that theorem. These require some further structure of the control system. We start with the case of a convex Hamiltonian

$$
H(x, p)=\max _{a \in A}\{-g(x, a) \cdot p\} .
$$

We define the following property of the the trajectories of the control system.

DEFINITION 4.9 We say that condition $\left(\mathrm{T}_{x}\right)$ holds at $x \in \Gamma$ if there are sequences $s_{n} \downarrow 0$ and $a_{n} \in L^{\infty}(0,+\infty ; A)$, a vector $\eta^{+}$pointing into $\Omega^{+}$and $k>0$ such that

$$
\begin{aligned}
& x_{n}=x\left(s_{n} ; x, a_{n}\right)=x+\left(s_{n}\right)^{k} \eta^{+}+o\left(\left(s_{n}\right)^{k}\right), \\
& x\left(0 ; x, a_{n}\right)=x .
\end{aligned}
$$

We have the following consequence.

PROPOSITION 4.10 If condition ( $\left.\mathrm{T}_{x}\right)$ holds at $x \in \Gamma$ then for any $t>0$, at $(x, t)$ we have

$$
\check{v}(x, t)=\lim _{n \rightarrow+\infty} \check{v}\left(x_{n}, t_{n}\right)
$$

where $\left.t_{n}=\check{t}\left(s_{n}\right)=t-\int_{0}^{s_{n}}\left(1 / f_{*}(x(s))\right) \mathrm{d} s, x_{n}=x\left(s_{n} ; a_{n}\right)=x+\left(s_{n}\right)^{k} \eta^{+}+o\left(\left(s_{n}\right)^{k}\right)\right)$, and $\eta^{+}$is a unit vector pointing into $\Omega^{+}$.

Proof. This is a consequence of the dynamic programming principle. Indeed, for any $s>0$ we have

$$
\check{v}(x, t)=\inf _{a \in L^{\infty}(0,+\infty ; A)} \check{v}\left(x\left(s \wedge \check{\tau}_{x, t}\right), t\left(s \wedge \check{\tau}_{x, t}\right)\right) .
$$

Therefore we immediately obtain, by choosing $s=s_{n}$ and $n$ sufficiently large,

$$
\check{v}(x, t) \leqslant \check{v}\left(x_{n}, t_{n}\right)
$$

and the conclusion follows since we already know from Remark 4.6 that $\breve{v}$ is an upper semicontinuous function.

We have obtained the following result.

THEOREM 4.11 Let $H$ be given as in (4.11). Assume $(2.4)$ and $(1.2)$ and suppose that condition $\left(\mathrm{T}_{x}\right)$ holds for all $x \in \Gamma$. Then the Cauchy problem 1.1 has a unique viscosity solution $v=\hat{v}=$ $\check{v} \in C\left(\mathbb{R}^{n} \times[0,+\infty)\right)$.

Proof. We use Corollary 3.1 with $\check{v}$ as subsolution and $\hat{v}$ as supersolution (notice that $t-\check{t}\left(s_{n}\right) \geqslant 0$ where $s_{n}$ appears in $\left(\mathrm{T}_{x}\right)$ ). We infer that $v=\hat{v}=\check{v}$ is a continuous solution of [1.1]. Then Corollary 3.5 states its uniqueness within discontinuous solutions.

EXAMPLE 4.12 Formula (4.12) is a very natural assumption. Suppose that for $x \in \Gamma$ there is $a \in A$ such that $n(x) \cdot g(x, a)>0$. Then we can choose $a_{n} \equiv a, s_{n}=1 / n, \eta=g(x, a)$, to reach condition $\left(\mathrm{T}_{x}\right)$ with $k=1$. 
Suppose that in a neighborhood of $x$ we have a symmetric system, i.e. for all $a \in A$ there is $\hat{a} \in A$ such that $g(x, a)=-g(x, \hat{a})$ and there is a Lie bracket such that the vector

$$
\eta^{+}=\left[g\left(\cdot, a_{1}\right), g\left(\cdot, a_{2}\right)\right](x)
$$

points into $\Omega^{+}$. Then we can satisfy ( $\mathrm{T}_{x}$ ) with $k=2$, by choosing $s_{n}=4 / n$,

$$
a_{n}(t)= \begin{cases}a_{1}, & s \in n^{-1}[0,1), \\ a_{2}, & s \in n^{-1}[1,2), \\ \hat{a}_{1}, & s \in n^{-1}[2,3), \\ \hat{a}_{2}, & s \in n^{-1}[3,4) .\end{cases}
$$

Indeed, for $k=2$, formula 4.12 is a basic expansion in differential geometry, explaining the role of the Lie bracket (see e.g. Chapter 5 in Spivak [23]).

The general structure (4.12) appears when similar higher order bracket conditions with respect to the Lie structure of the vector field $g$ are fulfilled as well. In fact (4.12), with an appropriate choice of $\eta^{+}$, is precisely the expansion of the trajectories as stated for instance in Haynes-Hermes [14]. For a more general proof of expansions like (4.12), an up to date discussion on this point and refinements, the reader can also check the recent work by Rampazzo-Sussmann [18].

We apply the example above to a specific, yet very interesting situation. Let $\sigma: \mathbb{R}^{n} \rightarrow \mathbb{R}^{n \times m}$ be a matrix valued function, whose columns are a family of $m(\leqslant n)$ Lipschitz continuous vector fields $\sigma_{i}: \mathbb{R}^{n} \rightarrow \mathbb{R}^{n}$, and then define an $n \times n$ positive semidefinite matrix valued function

$$
A(x)=\sigma(x) \sigma^{t}(x)
$$

whose quadratic form gives rise to the Hamiltonian

$$
H(x, p)=\sqrt{A(x) p \cdot p}=\left|\sigma^{t}(x) p\right|=\max _{|a| \leqslant 1}\{-\sigma(x) a \cdot p\} .
$$

In this case $g(x, a)=\sigma(x) a=\sum_{i=1}^{m} a_{i} \sigma_{i}(x), a=\left(a_{1}, \ldots, a_{m}\right)$ is the vector field of a symmetric control system. The corresponding HJ equation is the evolution eikonal equation

$$
u_{t}+f(x)\left|\sigma^{t}(x) D u\right|=0
$$

for an anisotropic medium with refraction index $1 / f$, subject to refraction since $f$ is discontinuous. When $A(\cdot)$ is positive definite, the Hamiltonian is coercive and the family of vector fields defines a Riemannian metric. When $A(\cdot)$ is degenerate but the Lie algebra generated by the family of vector fields $\left\{\sigma_{i}: i=1, \ldots, m\right\}$ satisfies the Hörmander condition (i.e. the dimension of $\operatorname{Lie}\left[\sigma_{1}, \ldots, \sigma_{m}\right]$ is $n$ at each point of the space) then the vector fields define a sub-Riemannian metric. In this case our HJ equation is of Carnot-Carathéodory type and the operator $\sigma^{t}(x) D u(x)=D_{h} u(x)$ is usually called the horizontal gradient of the function $u$. Condition $\left(\mathrm{T}_{x}\right)$ holds for instance at every point of $\Gamma$ (but this is far from being necessary) if the symmetric system satisfies the Hörmander condition just on $\Gamma$. A consequence is the following.

COROLlary 4.13 The Cauchy problem for the Carnot-Carathéodory eikonal equation with discontinuous refraction index

$$
\left\{\begin{array}{l}
u_{t}(x, t)+f(x)\left|D_{h} u(x, t)\right|=0 \\
u(x, 0)=u_{0}(x)
\end{array}\right.
$$


relative to a family of vector fields $\left\{\sigma_{i}(x): i=1, \ldots, m\right\}$ whose Lie algebra satisfies the Hörmander rank condition, has a continuous viscosity solution $\hat{v}=\breve{v}$.

Such a solution is unique in the class of discontinuous solutions and it is the uniform limit of solutions of approximating $\mathrm{HJ}$ equations obtained by approximating $f$ from below/above by continuous functions.

We now give an example of an existence and uniqueness theorem for nonconvex Hamiltonians as in (1.3). At $x \in \Gamma$, we consider the following assumption:

there is $a_{x} \in A$ such that $\mathcal{G}=\overline{\operatorname{co}} g\left(x, a_{x}, B\right) \cap\{0\}=\emptyset$

and for any $g \in \mathcal{G}, g /|g|$ is a unit vector pointing into $\Omega^{+}$.

$\left(\mathrm{TI}_{x}\right)$

EXAMPLE 4.14 The previous condition may look technical but it is very natural and easily satisfied. From the differential games point of view it means that at points in $\Gamma$ the controller $a$ can force the trajectories of the vector field $g(x, a, b)$ into $\Omega^{+}$by choosing $a(\cdot) \equiv a_{x}$.

In the case when $\Gamma$ is smooth, the condition simplifies and if $n_{x}^{+}$is the normal pointing into $\Omega^{+}$ at $x \in \Gamma$, by the Isaacs condition in (1.3) it can be rewritten explicitly on $H$ as

$$
H\left(x,-n_{x}^{+}\right)>0
$$

since we can find $a_{x} \in A$ such that

$$
H\left(x,-n_{x}^{+}\right)=\max _{a \in A} \min _{b \in B}\left\{-g(x, a, b) \cdot\left(-n_{x}^{+}\right)\right\}=\min _{b \in B}\left\{g\left(x, a_{x}, b\right) \cdot n_{x}^{+}\right\}=\min _{g \in \overline{\operatorname{co}} g\left(x, a_{x}, B\right)}\left\{g \cdot n_{x}^{+}\right\} .
$$

An example of a Hamiltonian in the plane that satisfies $\left(\mathrm{TI}_{x}\right)$ independently of the discontinuity set $\Gamma$ is any coercive (and nonconvex, homogeneous) function such as, for instance,

$$
H\left(x, y, p_{x}, p_{y}\right)=\left(2+4 \frac{p_{x} p_{y}\left(p_{x}^{2}-p_{y}^{2}\right)}{|p|^{4}}\right)|p| .
$$

Proposition 4.15 Assume 2.4 and (1.2). If condition $\left(\mathrm{TI}_{x}\right)$ holds at $x \in \Gamma$ then for any $t>0$, at $(x, t)$ we have

$$
\check{v}(x, t)=\lim _{n \rightarrow+\infty} \check{v}\left(x_{n}, t_{n}\right)
$$

for some sequence $\left(x_{n}, t_{n}\right)$ such that $t_{n} \leqslant t, x_{n}=x+s_{n} \eta^{+}+o\left(s_{n}\right)$ and $\eta^{+}$is a vector pointing into $\Omega^{+}$.

Proof. Given $(x, t), t>0$, by the dynamic programming principle for differential games (see e.g. [1]), for all $s>0$ sufficiently small we have

$$
\check{v}(x, t)=\inf _{\alpha \in \Delta} \sup _{b \in L^{\infty}(0,+\infty ; B)} \check{v}(x(s ; x, \alpha[b], b), \check{t}(s ; x, t, \alpha[b], b)) .
$$

Let now $x \in \Gamma$. By $\left(\mathrm{TI}_{x}\right)$ we define the strategy $\alpha[b] \equiv a_{x}$ and we choose a sequence $\left(x_{m}, t_{m}\right) \rightarrow(x, t)$ such that $\lim _{m \rightarrow+\infty} \check{v}\left(x_{m}, t_{m}\right)=\breve{v}^{*}(x, t)$. By 4.13), for any integer $m$ we select $b_{m} \in L^{\infty}(0, s ; B)$ such that

$$
\check{v}\left(x_{m}, t_{m}\right)-\frac{1}{m} \leqslant \breve{v}^{*}\left(x\left(s ; x_{m}, a_{x}, b_{m}\right), \check{t}\left(s ; x_{m}, t_{m}, a_{x}, b_{m}\right)\right) .
$$


Passing to a subsequence if necessary, we can find $b_{m} \stackrel{*}{\rightarrow} \hat{b} \in L^{\infty}(0, s ; P(B))$. By the properties of weak convergence it follows that $x\left(r ; x_{m}, a_{x}, b_{m}\right) \rightarrow x\left(r ; x, a_{x}, \hat{b}\right)$ for $r \in[0, s]$ where the latter trajectory solves the relaxed control system. Consequently, by Fatou's Lemma,

$$
\begin{aligned}
\liminf _{m \rightarrow+\infty} t\left(s ; x_{m}, t_{m}, a_{x}, b_{m}\right) & =\liminf _{m \rightarrow+\infty}\left(t_{m}-\int_{0}^{s} \frac{1}{f_{*}\left(x\left(s ; x_{m}, a_{x}, b_{m}\right)\right)} \mathrm{d} r\right) \\
& \geqslant t-\int_{0}^{s} \frac{1}{f_{*}\left(x\left(s ; x, a_{x}, \hat{b}\right)\right)} \mathrm{d} r .
\end{aligned}
$$

Moreover, as $\check{v}(x, \cdot)$ is nonincreasing, we deduce by passing to the limit in (4.14) that

$$
\check{v}^{*}(x, t) \leqslant \breve{v}^{*}\left(x\left(s ; x, a_{x}, \hat{b}\right), \check{t}\left(s ; x, t, a_{x}, \hat{b}\right)\right) .
$$

Now, for all $n \in \mathbb{N}$, we can select $0<s_{n} \leqslant 1 / n, x_{n}=x\left(s_{n} ; x, a_{x}, \hat{b}\right)$ and $t_{n}=\check{t}\left(s_{n} ; x, t, a_{x}, \hat{b}\right)$ (here $\hat{b}$ may depend on $n$ as well) so that

$$
\check{v}^{*}(x, t) \leqslant \check{v}^{*}\left(x_{n}, t_{n}\right) .
$$

In particular, by the upper semicontinuity of $\breve{v}^{*}$ we conclude that

$$
\check{v}^{*}(x, t)=\lim _{n \rightarrow+\infty} \check{v}^{*}\left(x_{n}, t_{n}\right) .
$$

To end the proof, observe that, by setting $g_{n}=\left(1 / s_{n}\right) \int_{0}^{s_{n}} g\left(x, a_{x}, \hat{b}(s)\right) \mathrm{d} s \in \cos g\left(x, a_{x}, B\right)$, we may suppose that at least along a subsequence, still denoted by $g_{n}, g_{n} \rightarrow \bar{g} \in \overline{\mathrm{co}} g\left(x, a_{x}, B\right)$. Now we use $\left(\mathrm{T}_{x}\right)$ again and observe that $|x(s)-x| \leqslant M_{g} s$ by 2.4 . Then we define $\eta^{+}=\bar{g} /|\bar{g}|$, a unit vector pointing into $\Omega^{+}$, and get

$$
\begin{aligned}
x_{n} & =x+\int_{0}^{s_{n}} g\left(x, a_{x}, \hat{b}(s)\right) \mathrm{d} s+o\left(s_{n}\right)=x+s_{n} g_{n}+o\left(s_{n}\right) \\
& =x+\left(s_{n}|\bar{g}|\right) \eta^{+}+o\left(s_{n}\right) \in \Omega^{+} .
\end{aligned}
$$

An easy consequence of our discussion is the existence and uniqueness statement.

THEOREM 4.16 Let $H$ be as in (1.3) such that the Isaacs condition holds. Assume (2.4) and (1.2) and suppose that condition $\left(\mathrm{TI}_{x}\right)$ holds for all $x \in \Gamma$. Then the Cauchy problem (1.1) has a unique viscosity solution $v=\hat{v}=\breve{v} \in C\left(\mathbb{R}^{n} \times[0,+\infty)\right)$.

Proof. We first use the comparison principle between $\hat{v}$ and $\check{v}^{*}$ to obtain

$$
\check{v}^{*} \leqslant \hat{v}\left(\leqslant \check{v}^{*}\right)
$$

and thus $\hat{v}$ is a continuous viscosity solution of 1.1 . Again by the comparison principle, $\hat{v}$ is the unique continuous solution.

\section{Acknowledgments}

Research of P. Soravia was partially supported by MIUR-Prin project "Metodi di viscosità, metrici e di teoria del controllo in equazioni alle derivate parziali nonlineari". 


\section{REFERENCES}

1. Bardi, M., \& CAPuzzo-DolcetTa, I. Optimal Control and Viscosity Solutions of Hamilton-JacobiBellman Equations. Birkhäuser (1997). Zbl 0890.49011 MR 1484411

2. BARDi, M., \& Soravia, P. A comparison result for Hamilton-Jacobi equations and applications to some differential games lacking controllability. Funkcial. Ekvac. 37 (1994), 19-43. Zbl 0823.49019 MR 1285479

3. Bonfiglioli, A., Lanconelli, E., \& Uguzzoni, F. Stratified Lie Groups and Potential Theory for Their Sub-Laplacians. Springer Monogr. Math., Springer, Berlin (2007). Zbl 1128.43001 MR 2363343

4. Caffarelli, L., Crandall, M. G., Kocan, M., \& Święch, A. On viscosity solutions of fully nonlinear equations with measurable ingredients. Comm. Pure Appl. Math. 49 (1996), 365-397. Zbl 0854.35032 MR 1376656

5. CAmilli, F. An Hopf-Lax formula for a class of measurable Hamilton-Jacobi equations. Nonlinear Anal. 57 (2004), 265-286. Zbl 1051.35029 MR 2056431

6. CAmilli, F., \& Siconolfi, A. Hamilton-Jacobi equations with measurable dependence on the state variable. Adv. Differential Equations 8 (2003), 733-768. Zbl 1036.35052 1969652

7. Chen, X. F., Hu, B. Viscosity solutions of discontinuous Hamilton-Jacobi equations. Interfaces Free Bound. 10 (2008), 339-359. Zbl 1162.35019 MR 2453135

8. Citti, G., \& SARTi, A. A cortical based model of perceptual completion in the roto-translation space. J. Math. Imaging Vision 24 (2006), 307-326. Zbl 1088.92008 MR 2235475

9. Crandall, M. G., Ishit, H., \& LiOns, P.-L. User's guide to viscosity solutions of second order partial differential equations. Bull. Amer. Math. Soc. 27 (1992), 1-67. Zbl $0755.35015 \mid$ MR 1118699

10. De Zan, C., \& Soravia, P. Singular limits for reaction diffusion equations and geometric flows with discontinuous velocity. Forthcoming.

11. Elliott, R. J., \& Kalton, N. J. The existence of value in differential games. Mem. Amer. Math. Soc. 126 (1972). Zbl 0262.90076 MR 0359845

12. Evans, L. C., \& Souganidis, P. E. Differential games and representation formulas for solutions of Hamilton-Jacobi-Isaacs equations. Indiana Univ. Math. J. 33 (1984), 773-797. Zbl 1169.91317 MR 0756158

13. GigA, Y. Surface Evolution Equations. A Level Set Approach. Monogr. Math. 99, Birkhäuser, Basel (2006). Zbl 1096.53039 MR 2238463

14. Haynes G. W., \& Hermes H. Nonlinear controllability via Lie theory. SIAM J. Control 8 (1970), 450-460. Zbl 0229.93012 MR 0277859

15. IsHII, H. Hamilton-Jacobi equations with discontinuous Hamiltonians on arbitrary open sets. Bull. Fac. Sci. Engrg. Chuo Univ. 28 (1985), 33-77. Zbl 0937.35505 MR 0845397

16. Jensen, R. Uniformly elliptic PDEs with bounded, measurable coefficients. J. Fourier Anal. Appl. 2 (1996), 237-259. Zbl 0890.35031 MR 1379505

17. Osher, S., \& Sethian, J. A. Fronts propagating with curvature-dependent speed: algorithms based on Hamilton-Jacobi formulations. J. Comput. Phys. 79 (1988), 12-49. Zbl 0659.65132 MR 0965860

18. RAMPAZZo, F., \& Sussmann, H. Set-valued differentials and a nonsmooth version of Chow's theorem. In: Proc. 40th IEEE Conference on Decision and Control (Orlando, FL, 2001), 2613-2618.

19. Soravia, P. Stability of dynamical systems with competitive controls: the degenerate case. J. Math. Anal. Appl. 191 (1995), 428-449. Zbl 0821.93059 MR 1325809

20. SoraviA, P. Boundary value problems for Hamilton-Jacobi equations with discontinuous Lagrangian. Indiana Univ. Math. J. 51 (2002), 451-476. Zbl 1032.35055 MR 1909297

21. Soravia, P. Degenerate eikonal equations with discontinuous refraction index. ESAIM Control Optim. Calc. Var. 12 (2006), 216-230. Zbl 1105.35026 MR 2209351 
22. SoraViA, P. Uniqueness results for viscosity solutions of fully nonlinear, degenerate elliptic equations with discontinuous coefficients. Comm. Pure Appl. Anal. 5 (2006), 213-240. Zbl 1132.35324 MR 2190786

23. SpIVAK, M. A Comprehensive Introduction to Differential Geometry, Vol. 1. Publish or Perish, Wilmington, DE (1979). Zbl 0439.53001 MR 0532830

24. Warga, J. Optimal Control of Differential and Functional Equations. Academic Press, New York (1972). Zbl 0253.49001 MR 0372708 